Supplement of Atmos. Chem. Phys. Discuss., 15, 34813-34869, 2015

http://www.atmos-chem-phys-discuss.net/15/34813/2015/

doi:10.5194/acpd-15-34813-2015-supplement

(C) Author(s) 2015. CC Attribution 3.0 License.

(c) (i)

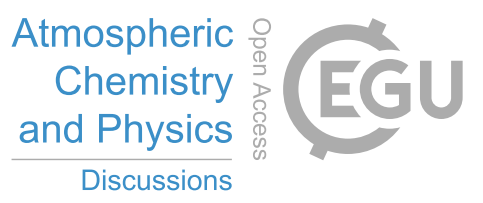

Supplement of

\title{
MIX: a mosaic Asian anthropogenic emission inventory for the MICS-Asia and the HTAP projects
}

M. Li et al.

Correspondence to: Q. Zhang (qiangzhang@ tsinghua.edu.cn)

The copyright of individual parts of the supplement might differ from the CC-BY 3.0 licence. 
Table S1. Monthly emissions by sector in Asia in 2010 (Units: Tg/month for $\mathrm{CO}_{2}$ and $\mathrm{Gg} / \mathrm{month}$ for other species).

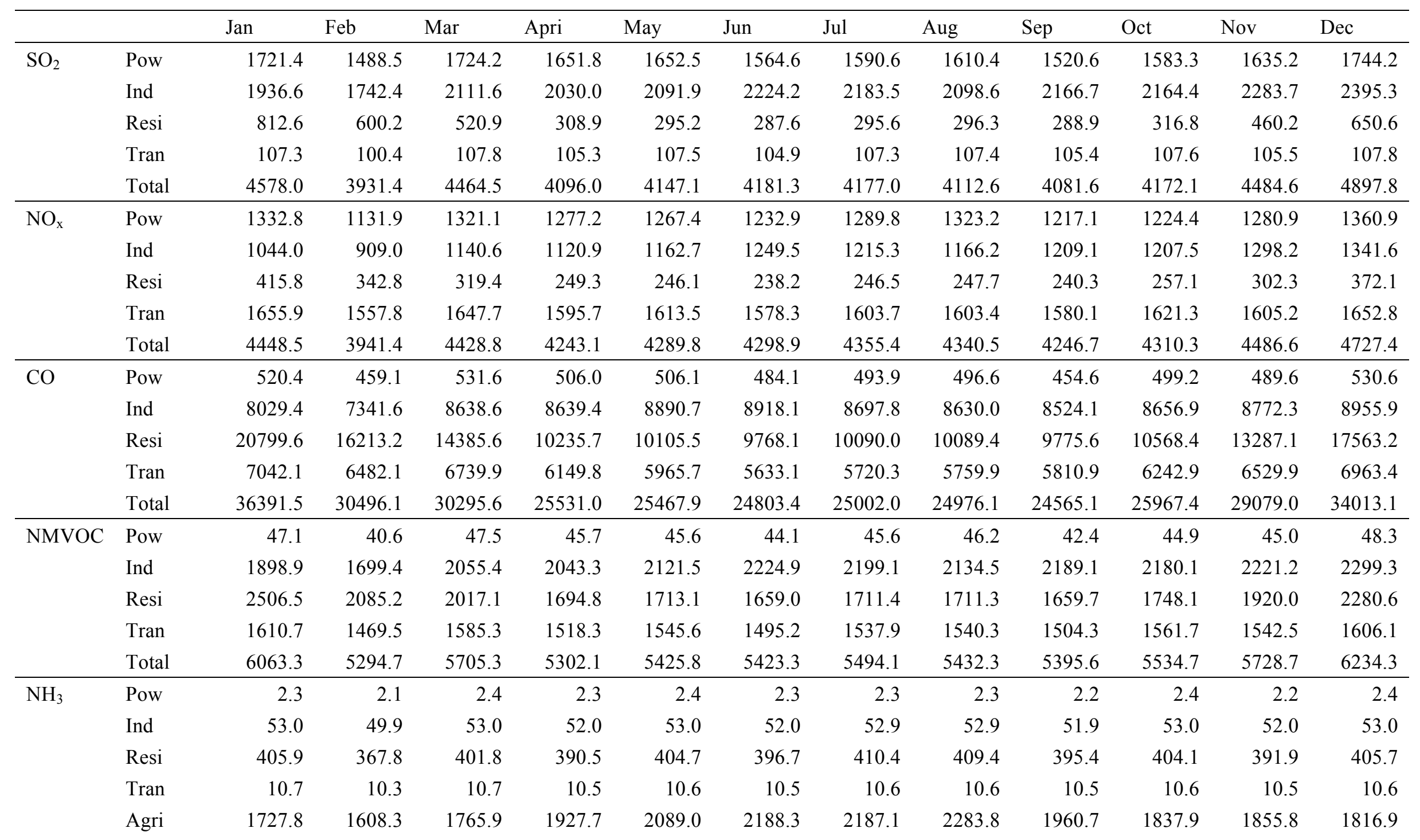




\begin{tabular}{|c|c|c|c|c|c|c|c|c|c|c|c|c|c|}
\hline & Total & 2199.6 & 2038.5 & 2233.7 & 2383.0 & 2559.6 & 2649.7 & 2663.3 & 2759.0 & 2420.7 & 2308.0 & 2312.3 & 2288.6 \\
\hline \multirow[t]{5}{*}{$\mathrm{PM}_{10}$} & Pow & 364.1 & 321.5 & 373.0 & 354.7 & 353.5 & 338.2 & 343.8 & 345.2 & 316.3 & 350.1 & 342.6 & 372.2 \\
\hline & Ind & 1155.8 & 1030.2 & 1253.5 & 1252.1 & 1295.6 & 1323.7 & 1297.2 & 1277.0 & 1288.7 & 1304.6 & 1340.0 & 1381.6 \\
\hline & Resi & 1379.3 & 1100.8 & 976.5 & 725.0 & 716.1 & 698.3 & 715.6 & 715.6 & 698.5 & 742.7 & 909.0 & 1173.5 \\
\hline & Tran & 158.5 & 146.0 & 154.6 & 148.3 & 148.8 & 144.3 & 147.1 & 147.5 & 145.7 & 151.2 & 150.8 & 157.7 \\
\hline & Total & 3057.8 & 2598.4 & 2757.6 & 2480.1 & 2513.9 & 2504.6 & 2503.7 & 2485.2 & 2449.2 & 2548.6 & 2742.3 & 3085.0 \\
\hline \multirow[t]{5}{*}{$\mathrm{PM}_{2.5}$} & Pow & 173.8 & 150.8 & 177.1 & 168.5 & 167.2 & 160.3 & 163.9 & 165.6 & 150.6 & 164.5 & 163.7 & 178.1 \\
\hline & Ind & 716.3 & 640.4 & 777.2 & 777.0 & 803.6 & 818.8 & 800.6 & 789.5 & 796.4 & 806.3 & 828.7 & 854.0 \\
\hline & Resi & 1253.3 & 1001.3 & 888.2 & 663.7 & 656.4 & 640.0 & 656.0 & 656.0 & 640.2 & 680.1 & 827.4 & 1067.3 \\
\hline & Tran & 152.1 & 140.1 & 148.2 & 142.3 & 142.7 & 138.4 & 141.0 & 141.4 & 139.7 & 145.0 & 144.7 & 151.4 \\
\hline & Total & 2295.5 & 1932.5 & 1990.7 & 1751.5 & 1769.9 & 1757.4 & 1761.4 & 1752.4 & 1726.9 & 1795.8 & 1964.5 & 2250.8 \\
\hline \multirow[t]{5}{*}{$\mathrm{BC}$} & Pow & 0.6 & 0.5 & 0.6 & 0.6 & 0.6 & 0.6 & 0.6 & 0.6 & 0.6 & 0.6 & 0.6 & 0.6 \\
\hline & Ind & 67.7 & 62.7 & 73.7 & 72.0 & 74.4 & 77.3 & 75.2 & 73.3 & 75.2 & 74.8 & 77.0 & 81.1 \\
\hline & Resi & 268.0 & 214.7 & 190.1 & 142.7 & 140.7 & 138.0 & 140.7 & 140.7 & 138.1 & 145.4 & 176.7 & 226.7 \\
\hline & Tran & 50.8 & 48.9 & 49.9 & 47.9 & 46.8 & 45.6 & 45.8 & 46.1 & 46.4 & 48.1 & 49.1 & 50.6 \\
\hline & Total & 387.2 & 326.8 & 314.4 & 263.2 & 262.6 & 261.6 & 262.3 & 260.7 & 260.3 & 268.8 & 303.5 & 359.1 \\
\hline \multirow[t]{5}{*}{$\mathrm{OC}$} & Pow & 1.1 & 1.0 & 1.1 & 1.0 & 1.1 & 1.0 & 1.0 & 1.0 & 1.0 & 1.1 & 1.0 & 1.1 \\
\hline & Ind & 94.5 & 87.2 & 100.2 & 98.2 & 101.3 & 101.8 & 100.7 & 99.5 & 99.9 & 100.6 & 101.7 & 106.0 \\
\hline & Resi & 850.7 & 692.5 & 617.1 & 480.2 & 475.4 & 466.0 & 475.2 & 475.2 & 466.1 & 489.6 & 578.8 & 731.2 \\
\hline & Tran & 28.7 & 27.1 & 28.0 & 26.6 & 26.0 & 25.0 & 25.2 & 25.4 & 25.5 & 26.8 & 27.5 & 28.6 \\
\hline & Total & 975.0 & 807.8 & 746.4 & 605.9 & 603.8 & 593.9 & 602.2 & 601.1 & 592.6 & 618.0 & 709.0 & 866.8 \\
\hline \multirow[t]{5}{*}{$\mathrm{CO}_{2}$} & Pow & 514.5 & 436.9 & 513.9 & 494.4 & 493.6 & 476.7 & 496.3 & 506.8 & 463.5 & 480.4 & 490.0 & 525.8 \\
\hline & Ind & 505.7 & 445.0 & 559.6 & 558.5 & 580.0 & 618.5 & 603.4 & 579.5 & 600.1 & 600.7 & 628.2 & 645.5 \\
\hline & Resi & 381.6 & 305.3 & 275.6 & 203.5 & 199.8 & 192.7 & 199.1 & 199.5 & 193.7 & 209.2 & 255.6 & 328.0 \\
\hline & Tran & 130.4 & 124.2 & 130.7 & 127.1 & 128.5 & 127.0 & 129.0 & 129.1 & 127.0 & 129.1 & 127.3 & 130.2 \\
\hline & Total & 1532.2 & 1311.4 & 1479.7 & 1383.5 & 1401.8 & 1414.9 & 1427.7 & 1415.0 & 1384.2 & 1419.4 & 1501.2 & 1629.6 \\
\hline
\end{tabular}


Table S2. Monthly emissions by Asian region in 2010 (Units: Tg/month for $\mathrm{CO}_{2}$ and $\mathrm{Gg} / \mathrm{month}$ for other species).

\begin{tabular}{|c|c|c|c|c|c|c|c|c|c|c|c|c|}
\hline Asia & Jan & Feb & Mar & Apri & May & Jun & Jul & Aug & Sep & Oct & Nov & Dec \\
\hline $\mathrm{SO}_{2}$ & 4578 & 3931 & 4464 & 4096 & 4147 & 4181 & 4177 & 4113 & 4082 & 4172 & 4485 & 4898 \\
\hline $\mathrm{NO}_{\mathrm{x}}$ & 4449 & 3941 & 4429 & 4243 & 4290 & 4299 & 4355 & 4340 & 4247 & 4310 & 4487 & 4727 \\
\hline $\mathrm{CO}$ & 36392 & 30496 & 30296 & 25531 & 25468 & 24803 & 25002 & 24976 & 24565 & 25967 & 29079 & 34013 \\
\hline NMVOC & 6063 & 5295 & 5705 & 5302 & 5426 & 5423 & 5494 & 5432 & 5396 & 5535 & 5729 & 6234 \\
\hline $\mathrm{NH}_{3}$ & 2200 & 2039 & 2234 & 2383 & 2560 & 2650 & 2663 & 2759 & 2421 & 2308 & 2312 & 2289 \\
\hline $\mathrm{PM}_{10}$ & 3058 & 2598 & 2758 & 2480 & 2514 & 2505 & 2504 & 2485 & 2449 & 2549 & 2742 & 3085 \\
\hline $\mathrm{PM}_{2.5}$ & 2295 & 1933 & 1991 & 1752 & 1770 & 1757 & 1761 & 1752 & 1727 & 1796 & 1965 & 2251 \\
\hline $\mathrm{BC}$ & 387 & 327 & 314 & 263 & 263 & 262 & 262 & 261 & 260 & 269 & 303 & 359 \\
\hline $\mathrm{OC}$ & 975 & 808 & 746 & 606 & 604 & 594 & 602 & 601 & 593 & 618 & 709 & 867 \\
\hline $\mathrm{CO}_{2}$ & 1532 & 1311 & 1480 & 1384 & 1402 & 1415 & 1428 & 1415 & 1384 & 1419 & 1501 & 1630 \\
\hline China & Jan & Feb & Mar & Apri & May & Jun & Jul & Aug & Sep & Oct & Nov & Dec \\
\hline $\mathrm{SO}_{2}$ & 2638 & 2144 & 2504 & 2225 & 2235 & 2336 & 2300 & 2244 & 2242 & 2262 & 2603 & 2931 \\
\hline $\mathrm{NO}_{\mathrm{x}}$ & 2460 & 2118 & 2458 & 2347 & 2360 & 2422 & 2432 & 2419 & 2367 & 2368 & 2578 & 2741 \\
\hline $\mathrm{CO}$ & 21428 & 17124 & 15857 & 11970 & 11796 & 11768 & 11630 & 11572 & 11390 & 12039 & 15157 & 19143 \\
\hline NMVOC & 2292 & 1911 & 2013 & 1751 & 1780 & 1895 & 1852 & 1791 & 1857 & 1867 & 2141 & 2468 \\
\hline $\mathrm{NH}_{3}$ & 610 & 606 & 646 & 836 & 941 & 1055 & 1005 & 1106 & 840 & 701 & 763 & 694 \\
\hline $\mathrm{PM}_{10}$ & 1794 & 1424 & 1465 & 1214 & 1224 & 1257 & 1235 & 1219 & 1222 & 1257 & 1505 & 1798 \\
\hline $\mathrm{PM}_{2.5}$ & 1416 & 1114 & 1094 & 869 & 870 & 886 & 874 & 865 & 863 & 895 & 1099 & 1355 \\
\hline $\mathrm{BC}$ & 232 & 181 & 164 & 119 & 117 & 119 & 118 & 116 & 117 & 122 & 156 & 204 \\
\hline $\mathrm{OC}$ & 543 & 404 & 330 & 201 & 194 & 192 & 194 & 193 & 189 & 207 & 300 & 437 \\
\hline $\mathrm{CO}_{2}$ & 913 & 744 & 862 & 790 & 799 & 830 & 829 & 820 & 808 & 810 & 911 & 1008 \\
\hline India & Jan & Feb & Mar & Apri & May & Jun & Jul & Aug & Sep & Oct & Nov & Dec \\
\hline $\mathrm{SO}_{2}$ & 799 & 753 & 822 & 769 & 781 & 747 & 742 & 736 & 740 & 774 & 775 & 822 \\
\hline $\mathrm{NO}_{\mathrm{x}}$ & 817 & 752 & 808 & 788 & 807 & 787 & 805 & 805 & 786 & 806 & 789 & 815 \\
\hline $\mathrm{CO}$ & 5872 & 5240 & 5727 & 5527 & 5688 & 5507 & 5656 & 5652 & 5475 & 5702 & 5535 & 5843 \\
\hline NMVOC & 1465 & 1306 & 1433 & 1382 & 1426 & 1380 & 1425 & 1423 & 1377 & 1428 & 1388 & 1458 \\
\hline $\mathrm{NH}_{3}$ & 840 & 758 & 838 & 811 & 838 & 811 & 838 & 838 & 811 & 838 & 811 & 840 \\
\hline $\mathrm{PM}_{10}$ & 613 & 573 & 616 & 593 & 600 & 581 & 584 & 580 & 560 & 602 & 577 & 614 \\
\hline $\mathrm{PM}_{2.5}$ & 451 & 421 & 448 & 433 & 439 & 427 & 432 & 430 & 418 & 441 & 426 & 450 \\
\hline $\mathrm{BC}$ & 87 & 86 & 86 & 84 & 84 & 84 & 84 & 84 & 84 & 84 & 85 & 87 \\
\hline $\mathrm{OC}$ & 217 & 213 & 211 & 209 & 209 & 209 & 209 & 209 & 209 & 209 & 210 & 215 \\
\hline $\mathrm{CO}_{2}$ & 197 & 180 & 200 & 191 & 195 & 186 & 187 & 186 & 177 & 195 & 184 & 197 \\
\hline $\begin{array}{ll}\text { Other } & \text { East } \\
\text { Asia } & \\
\end{array}$ & Jan & Feb & Mar & Apri & May & Jun & Jul & Aug & Sep & Oct & Nov & Dec \\
\hline $\mathrm{SO}_{2}$ & 120 & 114 & 120 & 119 & 119 & 119 & 122 & 121 & 120 & 123 & 119 & 121 \\
\hline $\mathrm{NO}_{\mathrm{x}}$ & 292 & 277 & 293 & 271 & 265 & 260 & 262 & 259 & 261 & 272 & 274 & 289 \\
\hline $\mathrm{CO}$ & 1005 & 910 & 970 & 853 & 827 & 768 & 771 & 764 & 768 & 842 & 885 & 976 \\
\hline NMVOC & 185 & 178 & 188 & 184 & 181 & 179 & 186 & 185 & 185 & 185 & 187 & 192 \\
\hline $\mathrm{NH}_{3}$ & 50 & 43 & 52 & 56 & 77 & 100 & 116 & 109 & 91 & 70 & 59 & 53 \\
\hline $\mathrm{PM}_{10}$ & 52 & 49 & 53 & 50 & 50 & 50 & 51 & 51 & 50 & 52 & 51 & 52 \\
\hline $\mathrm{PM}_{2.5}$ & 28 & 27 & 29 & 27 & 27 & 26 & 27 & 27 & 27 & 28 & 28 & 28 \\
\hline $\mathrm{BC}$ & 5 & 5 & 5 & 5 & 5 & 5 & 5 & 5 & 5 & 5 & 5 & 5 \\
\hline $\mathrm{OC}$ & 3 & 3 & 3 & 3 & 3 & 3 & 3 & 3 & 3 & 3 & 3 & 3 \\
\hline $\mathrm{CO}_{2}$ & 148 & 141 & 147 & 143 & 142 & 142 & 146 & 144 & 143 & 147 & 144 & 149 \\
\hline $\begin{array}{l}\text { Southeast } \\
\text { Asia }\end{array}$ & Jan & Feb & Mar & Apri & May & Jun & Jul & Aug & Sep & Oct & Nov & Dec \\
\hline
\end{tabular}




\begin{tabular}{|c|c|c|c|c|c|c|c|c|c|c|c|c|}
\hline $\mathrm{SO}_{2}$ & 377 & 340 & 377 & 366 & 376 & 366 & 379 & 379 & 366 & 378 & 366 & 378 \\
\hline $\mathrm{NO}_{\mathrm{x}}$ & 434 & 392 & 435 & 421 & 434 & 421 & 436 & 435 & 421 & 435 & 421 & 435 \\
\hline $\mathrm{CO}$ & 4340 & 3908 & 4320 & 4173 & 4309 & 4177 & 4322 & 4326 & 4184 & 4327 & 4195 & 4344 \\
\hline NMVOC & 1417 & 1278 & 1411 & 1364 & 1410 & 1366 & 1413 & 1413 & 1368 & 1414 & 1370 & 1417 \\
\hline $\mathrm{NH}_{3}$ & 390 & 352 & 390 & 378 & 390 & 377 & 390 & 390 & 377 & 390 & 377 & 390 \\
\hline $\mathrm{PM}_{10}$ & 257 & 229 & 258 & 253 & 259 & 252 & 260 & 260 & 250 & 260 & 250 & 263 \\
\hline $\mathrm{PM}_{2.5}$ & 192 & 172 & 193 & 189 & 194 & 188 & 194 & 194 & 186 & 194 & 187 & 196 \\
\hline $\mathrm{BC}$ & 32 & 29 & 32 & 31 & 32 & 31 & 32 & 32 & 31 & 32 & 31 & 32 \\
\hline $\mathrm{OC}$ & 123 & 111 & 123 & 119 & 123 & 119 & 123 & 123 & 119 & 123 & 119 & 124 \\
\hline $\mathrm{CO}_{2}$ & 129 & 116 & 130 & 126 & 129 & 126 & 130 & 130 & 125 & 130 & 126 & 130 \\
\hline $\begin{array}{l}\text { Other South } \\
\text { Asia }\end{array}$ & Jan & Feb & Mar & Apri & May & Jun & Jul & Aug & Sep & Oct & Nov & Dec \\
\hline $\mathrm{SO}_{2}$ & 146 & 131 & 144 & 140 & 144 & 140 & 144 & 144 & 140 & 144 & 140 & 146 \\
\hline $\mathrm{NO}_{\mathrm{x}}$ & 152 & 134 & 144 & 139 & 143 & 138 & 143 & 143 & 139 & 144 & 141 & 152 \\
\hline $\mathrm{CO}$ & 1544 & 1313 & 1348 & 1285 & 1320 & 1275 & 1316 & 1317 & 1279 & 1330 & 1330 & 1535 \\
\hline NMVOC & 361 & 312 & 327 & 313 & 323 & 312 & 322 & 322 & 312 & 324 & 321 & 359 \\
\hline $\mathrm{NH}_{3}$ & 294 & 264 & 291 & 282 & 291 & 282 & 291 & 291 & 282 & 291 & 282 & 294 \\
\hline $\mathrm{PM}_{10}$ & 120 & 103 & 108 & 103 & 106 & 103 & 106 & 106 & 103 & 107 & 106 & 119 \\
\hline $\mathrm{PM}_{2.5}$ & 102 & 87 & 90 & 86 & 89 & 86 & 89 & 89 & 86 & 89 & 89 & 101 \\
\hline $\mathrm{BC}$ & 19 & 16 & 17 & 16 & 16 & 16 & 16 & 16 & 16 & 16 & 16 & 19 \\
\hline $\mathrm{OC}$ & 67 & 57 & 58 & 55 & 57 & 55 & 57 & 57 & 55 & 57 & 57 & 67 \\
\hline $\mathrm{CO}_{2}$ & 38 & 33 & 35 & 34 & 35 & 34 & 35 & 35 & 34 & 35 & 34 & 38 \\
\hline Central Asia & Jan & Feb & Mar & Apri & May & Jun & Jul & Aug & Sep & Oct & Nov & Dec \\
\hline $\mathrm{SO}_{2}$ & 141 & 128 & 141 & 135 & 139 & 134 & 139 & 139 & 134 & 140 & 136 & 141 \\
\hline $\mathrm{NO}_{\mathrm{x}}$ & 86 & 78 & 83 & 79 & 80 & 77 & 80 & 80 & 78 & 81 & 81 & 86 \\
\hline $\mathrm{CO}$ & 593 & 542 & 512 & 404 & 365 & 320 & 326 & 328 & 357 & 424 & 478 & 578 \\
\hline NMVOC & 106 & 96 & 102 & 94 & 95 & 90 & 93 & 93 & 92 & 98 & 98 & 105 \\
\hline $\mathrm{NH}_{3}$ & 11 & 10 & 11 & 11 & 11 & 11 & 11 & 11 & 11 & 11 & 11 & 11 \\
\hline $\mathrm{PM}_{10}$ & 82 & 74 & 82 & 79 & 82 & 79 & 81 & 81 & 79 & 82 & 80 & 81 \\
\hline $\mathrm{PM}_{2.5}$ & 39 & 35 & 39 & 38 & 39 & 37 & 38 & 38 & 38 & 39 & 39 & 39 \\
\hline $\mathrm{BC}$ & 2 & 2 & 2 & 2 & 2 & 1 & 1 & 1 & 2 & 2 & 2 & 2 \\
\hline $\mathrm{OC}$ & 4 & 4 & 4 & 4 & 4 & 4 & 4 & 4 & 4 & 4 & 4 & 4 \\
\hline $\mathrm{CO}_{2}$ & 35 & 32 & 33 & 31 & 32 & 31 & 32 & 32 & 31 & 32 & 33 & 35 \\
\hline Russia Asia & Jan & Feb & Mar & Apri & May & Jun & Jul & Aug & Sep & Oct & Nov & Dec \\
\hline $\mathrm{SO}_{2}$ & 354 & 320 & 355 & 343 & 353 & 341 & 352 & 352 & 342 & 354 & 344 & 355 \\
\hline $\mathrm{NO}_{\mathrm{x}}$ & 209 & 189 & 209 & 199 & 201 & 192 & 197 & 198 & 194 & 205 & 202 & 209 \\
\hline $\mathrm{CO}$ & 1545 & 1408 & 1549 & 1337 & 1190 & 1011 & 1006 & 1044 & 1137 & 1326 & 1491 & 1562 \\
\hline NMVOC & 231 & 209 & 230 & 215 & 215 & 202 & 206 & 208 & 206 & 221 & 222 & 231 \\
\hline $\mathrm{NH}_{3}$ & 9 & 8 & 9 & 9 & 9 & 9 & 9 & 9 & 9 & 9 & 9 & 9 \\
\hline $\mathrm{PM}_{10}$ & 176 & 162 & 181 & 175 & 179 & 169 & 174 & 175 & 173 & 179 & 178 & 181 \\
\hline $\mathrm{PM}_{2.5}$ & 100 & 92 & 103 & 99 & 101 & 94 & 96 & 98 & 97 & 101 & 102 & 103 \\
\hline $\mathrm{BC}$ & 9 & 8 & 9 & 8 & 7 & 6 & 6 & 6 & 6 & 7 & 8 & 9 \\
\hline $\mathrm{OC}$ & 15 & 14 & 16 & 15 & 14 & 13 & 13 & 13 & 14 & 15 & 15 & 16 \\
\hline $\mathrm{CO}_{2}$ & 71 & 65 & 72 & 69 & 70 & 67 & 69 & 69 & 67 & 71 & 70 & 72 \\
\hline
\end{tabular}


Figure S1. Comparison of MIX and REAS2 estimates for China in 2008 by species and by sector.
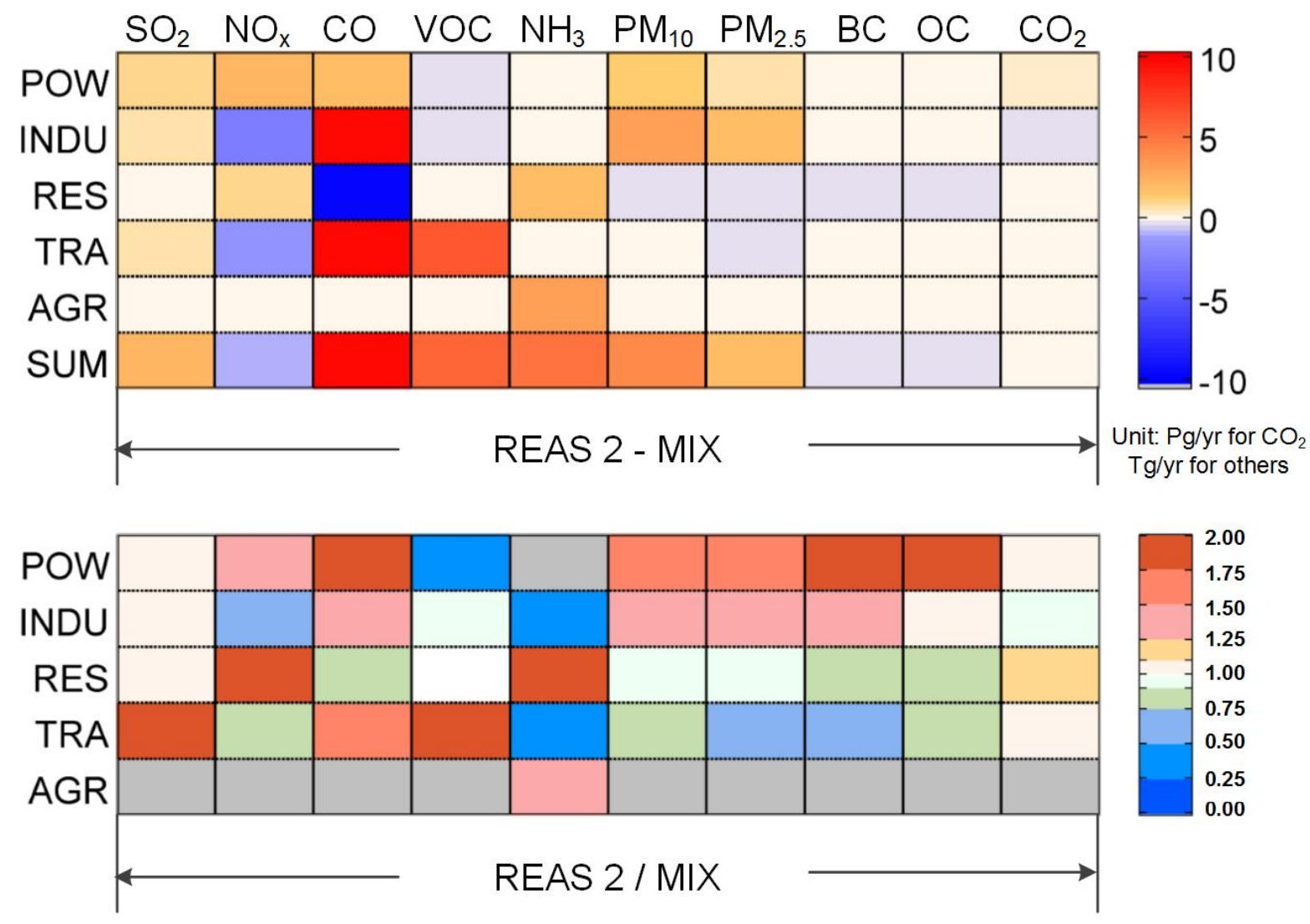